Originalien

Anaesthesist 2021 · 70:291-297

https://doi.org/10.1007/s00101-020-00888-2

Eingegangen: 16. Mai 2020

Überarbeitet: 18. Oktober 2020

Angenommen: 27. Oktober 2020

Online publiziert: 24. November 2020

(c) Der/die Autor(en) 2020

\author{
Johannes Schulz · Axel Scholler ${ }^{2}$ Paul Frank ${ }^{1}$ Dirk Scheinichen ${ }^{1}$. \\ Markus Flentje ${ }^{1} \cdot$ Hendrik Eismann $^{1} \cdot$ Thomas Palmaers $^{1}$ \\ ${ }^{1}$ Klinik für Anästhesiologie und Intensivmedizin (OE8050), Medizinische Hochschule Hannover, Hannover, \\ Deutschland \\ ${ }^{2}$ Anästhesiologische Klinik, Universitätsklinikum Erlangen, Erlangen, Deutschland
}

\title{
Komplikationen und Erfolgsraten des Vena-subclavia-Katheters in Abhängigkeit der Erfahrung
}

traschallgestützte Anlage eines ZVK zu favorisieren, dies noch nicht vollkommen umgesetzt ist. Für die Anlage eines ZVK via $\mathrm{V}$. jugularis interna verwendeten $80 \%$ der Befragten die ultraschallgesteuerte Methode. Bei der V. femoralis waren es nur $45 \%$ und bei der V. subclavia nur $39 \%$. Als Gründe wurden v.a. die fehlende Verfügbarkeit, eine verlängerte Prozedurenzeit und der Verlust an Fertigkeiten für die landmarkengestützte Variante genannt. Die ultraschallgestützte Punktion in „In-plane“-Technik ist anspruchsvoll und hat eine gewisse Lernkurve [10, 24]. Aber auch die landmarkengestützte Anlage des VSK hat mit Sicherheit eine Lernkurve. So konnten Maizel et al. [17] zeigen, dass junge Assistenzärzte, die die ultraschallgestützte Anlage eines ZVKerlernt hatten, eine hohe Misserfolgsquote und Komplikationsrate hatten, wenn sie aufgefordert wurden, eine landmarkengestützte Punktion durchzuführen. Aktuell gibt es in der verfügbaren Literatur keine ausreichenden Informationen, welchen Einfluss die Erfahrung eines/einer punktierenden Anästhesisten/Anästhesistin bei der landmarkengestützten Anlage eines VSK auf die Komplikationsraten und Erfolgsraten hat. Aus diesem Grund soll anhand einer Post-hoc-Analyse der Daten einer großen prospektiven Studie [25] der Einfluss der Punktionserfahrung bei der Anlage des VSK in Landmarkentechnik auf den Punktionserfolg und die Komplikationen ermittelt werden.

\section{Studiendesign und Untersuchungsmethoden}

Das Studiendesign und die Methode zur Randomisierung wurden bereits vorab veröffentlicht und werden hier erneut kurz dargestellt [25]:

Alle beschriebenen Untersuchungen am Menschen wurden mit Zustimmung der zuständigen Ethik-Kommission (Medizinische Hochschule Hannover 66242014, April 2014), im Einklang mit nationalem Recht sowie gemäß der Deklaration von Helsinki von 1975 (in der aktuellen, überarbeiteten Fassung) durchgeführt. Von allen beteiligten Patienten liegt eine schriftliche Einverständniserklärung vor, die vor Einschluss in die Studie und nach ausführlicher Aufklärung eingeholt wurde. Im Zeitraum von August 2014 und Oktober 2017 wurden insgesamt 1250 einschließbare Patienten in 2 deutschen großen Universitätskliniken identifiziert, von denen 1021 Patienten eingeschlossen wurden. Alle $\mathrm{Pa}$ tienten erhielten einen VSK, welcher in beiden Kliniken das Standardvorgehen für ZVK bei elektiven Kraniotomien ist.

Einschlusskriterien waren ein Alter von mindestens 18 Jahren sowie eine elektive Kraniotomie. Ausschlusskriterien waren anatomische Varianten an der Punktionsstelle, Zustand nach Klavikulafraktur, Infektionen an der Punktionsstelle und Schwangerschaft. In der ursprünglichen Fragestellung der Studienpopulation sollte untersucht werden, ob ein Unterschied in der Pneumothoraxrate vorliegt, abhängig davon, ob die Punk- 


\section{Originalien}

\begin{tabular}{|c|c|c|c|c|}
\hline Patientenalter (Jahre) & $M W \pm S D$ & $95 \%-K I$ & Minimum/Maximum & Gruppenunterschiede \\
\hline $0-20$ & $55,4 \pm 14,7$ & $53,9-56,8$ & $18-88$ & \multirow{3}{*}{$\begin{array}{l}0-20 \text { vs. } 21-50 ; p=1,000 \\
0-20 \text { vs. }>50 ; p=0,845 \\
21-50 \text { vs. }>50 ; p=1,000\end{array}$} \\
\hline $21-50$ & $56,3 \pm 15,9$ & $54,6-58,1$ & $18-87$ & \\
\hline$>50$ & $56,6 \pm 14,8$ & $54,9-58,3$ & $19-88$ & \\
\hline Patientengewicht $(\mathrm{kg})$ & $M W \pm S D$ & $95 \%-K I$ & Minimum/Maximum & Gruppenunterschiede \\
\hline $0-20$ & $78,6 \pm 17,3$ & $76,9-80,3$ & $44-158$ & \multirow{3}{*}{$\begin{array}{l}0-20 \text { vs. } 21-50 ; p=1,000 \\
0-20 \text { vs. }>50 ; p=0,851 \\
21-50 \text { vs. }>50 ; p=1,000\end{array}$} \\
\hline $21-50$ & $79,2 \pm 18,5$ & $77,7-81,2$ & $38-150$ & \\
\hline$>50$ & $80,1 \pm 18,6$ & $78,0-82,2$ & $39-183$ & \\
\hline Patientengröße ( $\mathrm{kg}$ ) & $M W \pm S D$ & $95 \%-K I$ & Minimum/Maximum & Gruppenunterschiede \\
\hline $0-20$ & $170 \pm 10$ & $169-171$ & $145-200$ & \multirow{3}{*}{$\begin{array}{l}0-20 \text { vs. } 21-50 ; p=1,000 \\
0-20 \text { vs. }>50 ; p=0,510 \\
21-50 \text { vs. }>50 ; p=1,000\end{array}$} \\
\hline $21-50$ & $171 \pm 10$ & $170-172$ & 150-199 & \\
\hline$>50$ & $171 \pm 10$ & $170-173$ & $150-198$ & \\
\hline Patienten, BMI $\left(\mathrm{kg} / \mathrm{m}^{2}\right)$ & $M W \pm S D$ & $95 \%-K I$ & Minimum/Maximum & Gruppenunterschiede \\
\hline $0-20$ & $27,0 \pm 5,5$ & $26,5-27,6$ & $17,7-61,7$ & \multirow{3}{*}{$\begin{array}{l}0-20 \text { vs. } 21-50 ; p=1,000 \\
0-20 \text { vs. }>50 ; p=1,000 \\
21-50 \text { vs. }>50 p=1,000\end{array}$} \\
\hline $21-50$ & $27,0 \pm 5,2$ & $26,3-27,5$ & $14,7-49,5$ & \\
\hline$>50$ & $27,2 \pm 5,9$ & $26,6-27,9$ & $14,3-56,5$ & \\
\hline Berufserfahrung (Jahre) & $M W \pm S D$ & $95 \%-K I$ & Minimum/Maximum & Gruppenunterschiede \\
\hline $0-20$ & $2,56 \pm 1,33$ & $2,43-2,69$ & $1-11$ & \multirow{3}{*}{$\begin{array}{l}0-20 \text { vs. } 21-50 ; p=0,002 \\
0-20 \text { vs. }>50 ; p<0,001 \\
21-50 \text { vs. }>50 ; p<0,001\end{array}$} \\
\hline $21-50$ & $4,09 \pm 2,91$ & $3,76-4,41$ & $1-18$ & \\
\hline$>50$ & $14,0 \pm 10,13$ & $12,84-15,13$ & $1-35$ & \\
\hline Geschlecht & Männlich;n (\%) & Weiblich; $n$ (\%) & - & Gruppenunterschiede \\
\hline $0-20$ & $168(37,4)$ & $232(40,6)$ & - & \multirow{3}{*}{$\begin{array}{l}0-20 \text { vs. } 21-50 ; p=0,909 \\
0-20 \text { vs. }>50 ; p=1,000 \\
21-50 \text { vs. }>50 ; p=1,000\end{array}$} \\
\hline $21-50$ & $144(32,1)$ & $170(29,7)$ & - & \\
\hline$>50$ & $137(30,5)$ & $170(29,7)$ & - & \\
\hline Punktionsbedingungen & Apnoe; $n(\%)$ & Beatmung; $n$ (\%) & - & Gruppenunterschiede \\
\hline $0-20$ & $193(39,7)$ & $207(38,7)$ & - & \multirow{3}{*}{$\begin{array}{l}0-20 \text { vs. } 21-50 ; p=1,000 \\
0-20 \text { vs. }>50 ; p=1,000 \\
21-50 \text { vs. }>50 ; p=1,000\end{array}$} \\
\hline $21-50$ & $152(31,3)$ & $162(30,3)$ & - & \\
\hline$>50$ & $141(29,0)$ & $166(31,0)$ & - & \\
\hline Studienzentrum & $\# 1 ; n(\%)$ & $\# 2 ; n(\%)$ & - & Gruppenunterschiede \\
\hline $0-20$ & $209(46,9)$ & $191(33,2)$ & - & \multirow{3}{*}{$\begin{array}{l}0-20 \text { vs. } 21-50 ; p=0,001 \\
0-20 \text { vs. }>50 ; p=0,018 \\
21-50 \text { vs. }>50 ; p<0,001\end{array}$} \\
\hline $21-50$ & $108(24,2)$ & $206(35,8)$ & - & \\
\hline$>50$ & $129(28,9)$ & $178(31,0)$ & - & \\
\hline
\end{tabular}

tion unter Apnoe oder fortgeführter Beatmung durchgeführt wird.

Nach der Randomisierung in die Interventionsgruppe Apnoe oder Beatmung begann die Einleitung einer Allgemeinanästhesie, und es erfolgte eine endotracheale Intubation. Die landmarkengestützte Punktion der V. subclavia wurde in normaler Rückenlage ohne Trendelenburg-Lagerung zur Vermeidung von Hirndruck durchgeführt. Beide Arme wurden angelagert und der Kopf in Neutralstellung positioniert. Auf Lagerungsmaterialien unter der Schulter oder Wirbelsäule wurde verzichtet. Die
Punktion erfolgte, außer bei Vorliegen von Kontraindikationen (ipsilateraler Portkatheter- oder Shunt-Verlauf, Axilladissektion etc.), in der Regel auf der rechten Seite.

Das Beatmungsgerät wurde zur Punktion entweder auf manuell/spontan (APL-Ventil 0 mbar) oder volumenkontrollierten Autoflow mit einem Tidalvolumen von $7 \mathrm{ml} / \mathrm{kg}$ idealem Körpergewicht (Körpergröße in Zentimetern minus 100) und einem positiven endexspiratorischen Druck (PEEP) von idealem Körpergewicht, geteilt durch 10 in mbar, eingestellt. Die Atemfre- quenz wurde entsprechend angepasst, um ein endexspiratorisches $\mathrm{CO}_{2}$ von $35-40 \mathrm{~mm} \mathrm{Hg}$ zu erreichen. Die Katheteranlagen wurden von anästhesiologischen Weiterbildungsassistent*innen mit mindestens einem Jahr Berufserfahrung oder Fachärzten/Fachärztinnen für Anästhesiologie durchgeführt. Die Punktion wurde grundsätzlich unter sterilen Bedingungen gemäß den RKI Richtlinien und in Seldinger-Technik [29] durchgeführt. Zur Anwendung kam ein $30 \mathrm{~cm}$ langer, 3-lumiger ZVK (Fa. Arrow International, Reading, PA, USA) mit 7,5 F. Es wurde zu keinem Zeitpunkt eine 
Anaesthesist 2021 · 70:291-297 https://doi.org/10.1007/s00101-020-00888-2

(c) Der/die Autor(en) 2020

J. Schulz · A. Scholler · P. Frank · D. Scheinichen $\cdot$ M. Flentje $\cdot$ H. Eismann $\cdot$ T. Palmaers

Komplikationen und Erfolgsraten des Vena-subclavia-Katheters in Abhängigkeit der Erfahrung

Zusammenfassung

Hintergrund. Die Punktion der V. subclavia gehört zu den Standardprozeduren eines/einer Anästhesisten/Anästhesistin. Gefürchtete Komplikationen dieser Prozedur sind der Pneumothorax und die arterielle Fehlpunktion. Zum Erlernen dieser Prozedur ist von einer gewissen Lernkurve auszugehen. Ziel der Arbeit. In dieser Studie soll der Einfluss der Punktionserfahrung auf die Erfolgsquote und mechanische Komplikationen wie Pneumothorax und arterielle Punktion untersucht werden. Dazu sollen 3 Erfahrungsstufen miteinander verglichen werden: unerfahren: 0 bis 20 Punktionen, mäßig erfahren: 21 bis 50 und erfahren: über 50 Punktionen.
Material und Methoden. Post-hoc-Analyse einer vorab publizierten Nichtunterlegenheitsstudie zur Untersuchung des Einflusses der Beatmung auf die Pneumothoraxrate bei der V.-subclavia-Punktion in Landmarkentechnik. Es wurden 1021 Patienten ausgewertet, die in die vorab publizierte Studie zwischen August 2014 und Oktober 2017 eingeschlossen wurden.

Ergebnisse. Die Gesamtrate an mechanischen Komplikationen ist in der Gruppe der Unerfahrenen im Vergleich zur Gruppe der Erfahrenen signifikant höher ( $15 \%$ vs. $8,5 \%, p=0,023$ ).

Ebenso ist die Rate an Punktionsversuchen in der Gruppe der Unerfahrenen (0-20) mit $1,85 \pm 1,12$ signifikant höher als in der Gruppe der Erfahrenen $(1,58 \pm 0,99, p=0,004)$. Im Gegenzug war die Rate einer erfolgreichen Punktion mit 95,1\% in der Gruppe der Erfahrenen signifikant höher als in der Gruppe der Unerfahrenen $(89,3 \%, p=0,001)$.

Schlussfolgerung. Für eine erfolgreiche Punktion der V. subclavia in Landmarkentechnik ist eine Lernkurve von mindestens 50 Punktionen nötig, um die Komplikationsrate zu senken und die Erfolgsrate zu steigern.

Schlüsselwörter

Zentraler Venenkatheter - Intensivmedizin . Lernkurve · Pneumothorax · Ausbildung

\section{Complications and success rates of subclavian vein catheterization depending on experience}

\section{Abstract}

Background. The infraclavicular puncture of the subclavian vein is a standard procedure for anesthetists. Meanwhile the literature and recommendations are clear and the use of real-time ultrasound guidance is the standard procedure; however, anesthetists will always get into special circumstances were they have to use the landmark technique, so this competence must be preserved. Feared complications of infraclavicular subclavian vein puncture are pneumothorax and arterial puncture. Up to now there is no clear learning curve for the infraclavicular subclavian vein puncture in the landmark technique performed by anesthetists.

Objective. The aim of this study was to examine the influence of the puncture experience on the success rate and mechanical complications, such as pneumothorax and arterial puncture in patients who received an infraclavicular subclavian vein puncture with the landmark technique. Three levels of experience were defined for comparison: inexperienced $0-20$ punctures, moderately experienced $21-50$ and experienced over 50 punctures.

Material and methods. Post hoc analysis of a previously published noninferiority study to examine the influence of ventilation on the pneumothorax rate in the subclavian vein puncture using the landmark technique. This analysis included 1021 anesthetized patients who were included in the original study between August 2014 and October 2017. Demographic data as well as the number of puncture attempts, puncture success, the overall rate of mechanical complications, pneumothorax rate and arterial puncture rates were calculated.

Results. The overall rate of mechanical complications (pneumothorax + arterial puncture) was significantly higher in the inexperienced group (0-21) compared to the experienced group ( $>50,15 \%$ vs. $8.5 \%$, respectively, $p=0.023)$. This resulted in an odds ratio of 0.52 (confidence interval, Cl: $0.32-0.85$, $p=0.027$ ). Likewise, the rate of puncture attempts in the group of inexperienced $(0-20)$ with $1.85 \pm 1.12$ was significantly higher than in the group of experienced $(>50$, $1.58 \pm 0.99, p=0.004)$ and resulted in an odds ratio of 0.59 (Cl: $0.31-0.96, p=0.028$ ). Although the puncture attempts of the moderately experienced (21-50) compared to the inexperienced (0-20) was not significant lower, we found an odds ratio of 0.69 (Cl:
$0.48-0.99, p=0.042)$. The rate of successful puncture was $95.1 \%$ in the experienced group versus $89.3 \%$ in the inexperienced group ( $p=0.001)$, which resulted in an odds ratio of 2.35 (Cl: $1.28-4.31, p=0.018)$. When viewed individually, no significant differences were found for pneumothorax and arterial puncture.

Conclusion. In this post hoc analysis of the puncture of the subclavian vein using the landmark technique, we found a significant reduction of puncture attempts and overall mechanical complications. At least 50 punctures seem to be necessary to achieve the end of the learning curve; however, the landmark technique should only be used under special circumstances, when realtime ultrasound is not available. Anesthetists who want to complete their repertoire and learn the landmark technique should always perform a static ultrasound examination before starting the puncture in order to reduce complications due to anatomical variations or thrombosis.

Keywords

Central venous catheter - Intensive care .

Learning curve $\cdot$ Pneumothorax - Education 
Tab. 2 Komplikationen in Abhängigkeit von der Punktionserfahrung

\begin{tabular}{|c|c|c|c|}
\hline Komplikationen & $J a ; n(\%)$ & Nein;n (\%) & Gruppenunterschiede \\
\hline $0-20$ & $60(15)$ & $340(85)$ & \multirow{3}{*}{$\begin{array}{l}0-20 \text { vs. } 21-50 ; p=0,340 \\
0-20 \text { vs. }>50 ; \boldsymbol{p}=0,023 \\
21-50 \text { vs. }>50 p=0,940\end{array}$} \\
\hline $21-50$ & $35(11,1)$ & $279(88,9)$ & \\
\hline$>50$ & $26(8,5)$ & $281(91,5)$ & \\
\hline Punktionserfolg & $J a ; n(\%)$ & Nein;n (\%) & Gruppenunterschiede \\
\hline $0-20$ & $357(89,3)$ & $43(10,7)$ & \multirow{3}{*}{$\begin{array}{l}0-20 \text { vs. } 21-50 ; p=0,054 \\
0-20 \text { vs. }>50 ; \boldsymbol{p}=0,001 \\
21-50 \text { vs. }>50 ; p=1,000\end{array}$} \\
\hline $21-50$ & $295(93,9)$ & $19(6,1)$ & \\
\hline$>50$ & $292(95,1)$ & $15(4,9)$ & \\
\hline Pneumothorax & $J a ; n(\%)$ & Nein; $n(\%)$ & Gruppenunterschiede \\
\hline $0-20$ & $5(1,3)$ & $395(98,7)$ & \multirow{3}{*}{$\begin{array}{l}0-20 \text { vs. } 21-50 ; p=0,416 \\
0-20 \text { vs. }>50 ; p=0,883 \\
21-50 \text { vs. }>50 ; p=0,052\end{array}$} \\
\hline $21-50$ & $8(2,5)$ & $306(97,5)$ & \\
\hline$>50$ & $1(0,3 \%)$ & $306(99,7 \%)$ & \\
\hline Arterielle Punktion & $J a ; n(\%)$ & Nein; $n(\%)$ & Gruppenunterschiede \\
\hline $0-20$ & $30(7,5)$ & $370(92,5)$ & \multirow{3}{*}{$\begin{array}{l}0-20 \text { vs. } 21-50 ; p=0,254 \\
0-20 \text { vs. }>50 ; p=0,283 \\
21-50 \text { vs. }>50 ; p=1,000\end{array}$} \\
\hline $21-50$ & $14(4,5)$ & $300(95,5)$ & \\
\hline$>50$ & $14(4,6)$ & $293(95,4)$ & \\
\hline Punktionsversuche & $M W \pm S D($ Min/Max $)$ & $95 \%-K I$ & Gruppenunterschiede \\
\hline $0-20$ & $1,85 \pm 1,12(1-6)$ & $1,73-1,93$ & \multirow{3}{*}{$\begin{array}{l}0-20 \text { vs. } 21-50 ; p=0,203 \\
0-20 \text { vs. }>50 ; \boldsymbol{p}=0,004 \\
21-50 \text { vs. }>50 ; p=0,526\end{array}$} \\
\hline $21-50$ & $1,69 \pm 1,11(1-6)$ & $1,57-1,82$ & \\
\hline$>50$ & $1,58 \pm 0,99(1-6)$ & $1,47-1,69$ & \\
\hline \multicolumn{4}{|c|}{$\begin{array}{l}\text { Gesamtrate an Komplikationen (Erfolglosigkeit, Pneumothorax und arterielle Fehlpunktion) sowie } \\
\text { Einzelbetrachtung der Komplikationen, abhängig von der Punktionserfahrung (Anzahl der bisher } \\
\text { durchgeführten Subclavia-Anlagen) in den } 3 \text { Kategorien. Anzahl und prozentualer Anteil sind als } \\
n \text { (\%) ausgewiesen. MW stellt den Mittelwert dar, SD die Standardabweichung und } 95 \% \text {-KI das } \\
95 \% \text {-Konfidenzintervall der Mittelwerte. Min Minimum, Max Maximum. Gruppenunterschiede } \\
\text { wurden mittels ANOVA mit Bonferroni-Post-hoc-Test ermittelt. } p<0,05 \text { statistisch signifikant (fett) }\end{array}$} \\
\hline
\end{tabular}

Ultraschallunterstützung durchgeführt. Die Punktionstechnik war standardisiert. Die Punktionsstelle wurde ca. $1 \mathrm{~cm}$ infraklavikulär am Übergang des medialen zum lateralen Drittel der Klavikula aufgesucht und in Richtung Klavikula vorgeschoben, bis Knochenkontakt spürbar war. Anschließend wurde die Nadel zum Jugulum ausgerichtet, und es wurde unter Aspiration vorgeschoben. Bei Erfolglosigkeit wurde die Nadel bis auf das Hautniveau zurückgezogen, und ein neuer Punktionsversuch mit kranialerer Stichrichtung wurde unternommen. War auch dieser nicht erfolgreich, wurde die Nadel erneut auf Hautniveau zurückgezogen und kaudaler als bei dem ersten Versuch ausgerichtet. Waren alle 3 Punktionsversuche erfolglos, musste die Punktion an eine/n zweite/n Anästhesisten/Anästhesistin mit fachärztlicher Qualifikation übergeben werden. Dieser durfte ebenfalls maximal 3 Punktionsversuche unternehmen, nach dem oben beschriebenen Verfahren. Nach maximal 6 erfolglosen Versuchen wurde
Nach erfolgreicher Punktion wurde die korrekte Katheterlage mittels intrakardialem EKG und Aspirationsprobe an allen Lumina verifiziert. Eine arterielle Fehllage wurde durch eine venöse Rücklaufprobe ausgeschlossen.

Postoperativ erhielten die Patienten zum Ausschluss bzw. Nachweis eines Pneumothorax eine Sonographie des Thorax. Dazu wurde in 4 Quadranten mittels Seashore-Zeichen oder BarcodeZeichen im M-Mode der Pneumothorax ausgeschlossen bzw. verifiziert [1]. Bei positivem Bar-Code-Zeichen wurde eine Thoraxröntgenaufnahme angefertigt, um die Größe und Interventionspflichtigkeit zu bestimmen.

\section{Statistische Analyse und Fallzahlplanung}

Statistische Analysen wurden mit Stata 15.1 (StataCorp LLC, Texas, USA) und SPSS 22 (IBM Deutschland, Ehingen, Germany) durchgeführt.

Alle Variablen wurden mit dem Shapiro-Wilks-Test auf Normalverteilung getestet. Normal verteilte Daten werden als Mittelwerte mit Standardabweichung und Minimum und Maximum dargestellt. Gruppenunterschiede wurden mittels $\mathrm{t}$-Test für nichtverbundene Stichproben ausgewertet bzw. mittels einer ANOVA mit folgendem Bonferroni-Post-hoc-Test. Dichotome Variablen sind als Häufigkeiten und prozentuale Anteile dargestellt. Unterschiede wurden ebenfalls mittels ANOVA mit folgendem Bonferroni-Post-hoc-Test ermittelt. Die multivariate logistische Regressionsanalyse wurde angewendet, um die „oddsratio“ mit zugehörigen $95 \%$-Konfidenzintervallen für Komplikationsraten (einzeln und insgesamt) sowie den Punktionserfolg, adjustiert für die in 3 Stufen kategorisierte Punktionserfahrung, zu ermitteln. Ein $p$-Wert $<0,05$ wurde in allen Testverfahren als statistisch signifikant angenommen.

\section{Ergebnisse}

In diese Post-hoc-Analyse wurden 1021 Patienten eingeschlossen. Es traten insgesamt 14 Pneumothoraces auf (1,4\%), von denen 9 mittels Thorax- 


\begin{tabular}{|c|c|c|c|}
\hline Komplikationen & Odds ratio & $95 \%-K o n f i d e n z i n t e r v a l l$ & - \\
\hline $21-50$ vs. $0-20$ & 0,71 & $0,46-1,11$ & $p=0,402$ \\
\hline$>50$ vs. $0-20$ & 0,52 & $0,32-0,85$ & $p=0,027$ \\
\hline$>50$ vs. $21-50$ & 0,74 & $0,43-1,26$ & $p=0,792$ \\
\hline Punktionserfolg & Odds ratio & $95 \%-K o n f i d e n z i n t e r v a l l$ & - \\
\hline $21-50$ vs. $0-20$ & 1,87 & $1,07-3,28$ & $p=0,087$ \\
\hline$>50$ vs. $0-20$ & 2,35 & $1,28-4,31$ & $p=0,018$ \\
\hline$>50$ vs. $21-50$ & 1,25 & $0,63-2,52$ & $p=1,000$ \\
\hline Punktionsversuche & Odds ratio & 95\%-Konfidenzintervall & - \\
\hline $21-50$ vs. $0-20$ & 0,69 & $0,48-0,99$ & $p=0,042$ \\
\hline$>50$ vs. $0-20$ & 0,59 & $0,37-0,96$ & $p=0,028$ \\
\hline$>50$ vs. $21-50$ & 0,86 & $0,54-1,39$ & $p=1,000$ \\
\hline Arterielle Punktion & Odds ratio & 95\%-Konfidenzintervall & - \\
\hline $21-50$ vs. $0-20$ & 0,58 & $0,30-1,11$ & $p=0,291$ \\
\hline$>50$ vs. $0-20$ & 0,56 & $0,31-1,13$ & $p=0,336$ \\
\hline$>50$ vs. $21-50$ & 1,02 & $0,48-2,19$ & $p=1,000$ \\
\hline Pneumothorax & Odds ratio & $95 \%$-Konfidenzintervall & - \\
\hline $21-50$ vs. $0-20$ & 2,07 & $0,67-6,38$ & $p=0,621$ \\
\hline$>50$ vs. $0-20$ & 0,26 & $0,03-2,22$ & $p=0,654$ \\
\hline$>50$ vs. $21-50$ & 0,13 & $0,02-1,00$ & $p=0,153$ \\
\hline \multicolumn{4}{|c|}{$\begin{array}{l}p<0,05 \text { statistisch signifikant (fett) } \\
\text { Chancenverhältnisse (Odds ratio) mit zugehörigen Konfidenzintervallen und Bonferroni-korrigierte } \\
\text { Signifikanzniveaus ( } p \text { ) für Komplikationen insgesamt sowie für die einzelnen Komplikationen und } \\
\text { Erfolgsraten, abhängig von der Punktionserfahrung in den } 3 \text { Kategorien }\end{array}$} \\
\hline
\end{tabular}

drainage entlastet werden mussten. Es trat bzw. traten kein Hämatothorax oder sonstige schwere mechanische Komplikationen auf. Die Gesamtkomplikationsrate der gesamten Population betrug $11,9 \%$, davon entfielen $5,7 \%$ auf arterielle Punktionen. In 92,5\% der Fälle war die Punktion erfolgreich. Die demografischen Daten der Patienten sind in - Tab. 1 dargestellt. Es gab in der Studienpopulation innerhalb der 3 Kategorien für die Punktionserfahrung keinen signifikanten Unterschied bezüglich Alter, Gewicht, Größe, BMI oder Geschlecht. Auch waren die Patienten bezüglich der 3 Erfahrungskategorien in beiden Interventionsgruppen homogen verteilt.

In der Verteilung der Punktionserfahrung sowie der Berufserfahrung zeigte sich für alle 3 Kategorien ein signifikanter Unterschied (• Tab. 1): Die Kolleg*innen mit den meisten Punktionen hatten auch mehr Berufserfahrung. Die Komplikationsrate insgesamt war mit $15 \%$ in der Gruppe mit 0-20 VSK signifikant höher als in der Gruppe mit mindestens 50 VSK (8,5\%). Auch beim Punktionserfolg gab es zwischen den
Unerfahrenen und Erfahrenen einen signifikanten Unterschied $(89,3 \%$ vs. 95,1\%) (• Tab. 2). Zwischen den Gruppen $>50$ VSK vs. 21-50 VSK waren diese Unterschiede nicht mehr signifikant. Beim Vergleich der Komplikationsentitäten arterielle Punktion und Pneumothorax konnten keine signifikanten Unterschiede aufgezeigt werden. Bei der Anzahl der benötigten Punktionsversuche war die Gruppe mit einer Erfahrung von mindestens 50 VSK der Gruppe mit nur 0-20 VSK signifikant überlegen $(1,58 \pm 0,99$ vs. $1,85 \pm 1,12, p<0,01)$ (• Tab. 2).

Dies spiegelt sich in einer 2,35fach höheren Wahrscheinlichkeit $(p=0,018)$ wider, eine Punktion erfolgreich abzuschließen, wenn man im Vergleich zur Gruppe mit unter 20 VSK bereits mehr als 50 VSK-Anlagen durchgeführt hat (- Tab. 3). Auch die Odds ratios für Punktionsversuche sind signifikant niedriger für die beiden erfahreneren Gruppen gegenüber 0-20 VSK-Anlagen (OR 0,59 für $>50$ VSK-Anlagen vs. $0-20$ und 0,69 für 21-50 VSK-Anlagen 0-20 (jeweils $p<0,05))$.
In einer Subgruppenanalyse der Erstpunktierenden (Punktionen 1 bis max. 3) zeigte sich eine signifikante Erhöhung des Punktionserfolges, abhängig von der Punktionserfahrung (• Tab. 4).

\section{Diskussion}

Mit dieser Auswertung konnten aufgezeigt werden, dass die erfolgreiche Anlage eines VSK in Landmarkentechnik maßgeblich von der Punktionserfahrung abhängig ist. Demzufolge haben erfahrene Anästhesieärzte/-ärztinnen mit mind. 50 VSK-Anlagen eine signifikant höhere Erfolgsquote als unerfahrene Ärzte/ Ärztinnen (bis zu 20 VSK). Gleichzeitig fallen die Komplikationsraten und die Anzahl der Punktionsversuche von erfahrenen gegenüber unerfahrenen Ärzten/Ärztinnen signifikant geringer aus. Diese Ergebnisse stehen im Einklang mit Daten von Sznajder et al., die ebenfalls nachweisen konnten, dass sich der Punktionserfolg erst nach über 50 durchgeführten Prozeduren signifikant verbessert [31]. Mansfield et al. konnten zeigen, dass steigende Erfahrung in Berufsjahren bei der Anlage eines VSK zu einer signifikanten Verbesserung der Erfolgsquote führt [18]. Im Kontrast zu den genannten Studien konnten Lefrant et al. keinen Zusammenhang zwischen Erfahrung und dem Punktionserfolg oder der Komplikationsrate sehen [16]. Dies steht im Gegensatz zu diesen Ergebnissen, die eine signifikant niedrigere Gesamtkomplikationsrate bei einer Erfahrung von mindestens 50 Punktionen aufzeigt. Der positive Einfluss der Berufserfahrung auf den Punktionserfolg und die Komplikationsrate konnte auch von anderen Autoren aufgezeigt werden [7, 9, 22]. In diesen Studien wurde die Berufserfahrung über den Ausbildungsstand der punktierenden Ärzte/Ärztinnen definiert. Auch in dieser Untersuchung zeigte sich im Vergleich der beiden Studienzentren die Relevanz des Ausbildungsstandes. Im Zentrum \#1 war die Gruppe der unerfahrenen Ärzte/Ärztinnen im Vergleich zum Zentrum \#2 deutlich größer (33,2\% vs. $46,9 \%)$; dies zeigte sich in den vorab publizierten Ergebnissen entsprechend in den Komplikationsraten der Studienzentren [25]. 
Tab. 4 Punktionserfolg und Punktionsversuche bis maximal 3 Punktionsversuche (Subgruppenanalyse, $n=928$ )

\begin{tabular}{|c|c|c|c|}
\hline Punktionserfolg & $J a ; n(\%)$ & Nein;n (\%) & Gruppenunterschiede \\
\hline $0-20$ & $331(82,8)$ & $69(17,3)$ & \multirow{3}{*}{$\begin{array}{l}0-20 \text { vs. } 21-50 ; p<0,001 \\
0-20 \text { vs. }>50 ; p<0,001 \\
21-50 \text { vs. }>50 ; p<0,001\end{array}$} \\
\hline $21-50$ & $275(87,6)$ & $39(12,4)$ & \\
\hline$>50$ & $278(90,6)$ & $29(9,4)$ & \\
\hline Punktionsversuche & $M W \pm S D(M i n / M a x)$ & $95 \%-K l$ & Gruppenunterschiede \\
\hline $0-20$ & $1,68 \pm 0,83(1-3)$ & $1,60-1,76$ & \multirow{3}{*}{$\begin{array}{l}0-20 \text { vs. } 21-50 ; p=0,147 \\
0-20 \text { vs. }>50 ; \boldsymbol{p}=0,003 \\
21-50 \text { vs. }>50 ; p=0,590\end{array}$} \\
\hline $21-50$ & $1,56 \pm 0,78(1-3)$ & $1,47-1,65$ & \\
\hline$>50$ & $1,48 \pm 0,73(1-3)$ & $1,40-1,56$ & \\
\hline Punktionserfolg & Odds ratio & $95 \%-K I$ & - \\
\hline $21-50$ vs. $0-20$ & 1,47 & $0,96-2,25$ & $p=0,075$ \\
\hline$>50$ vs. $0-20$ & 2,00 & $1,26-3,17$ & $p=0,006$ \\
\hline$>50$ vs. $21-50$ & 1,36 & $0,82-2,26$ & $p=0,237$ \\
\hline \multicolumn{4}{|c|}{$\begin{array}{l}\text { Subgruppenanalyse der Erstpunktierenden (maximal } 3 \text { Punktionen, bevor der Punkteur gewechselt } \\
\text { wurde) bezüglich Erfolglosigkeit und Punktionsversuchen, abhängig von der Punktionserfahrung } \\
\text { (Anzahl der bisher durchgeführten Subclavia-Anlagen) in den } 3 \text { Kategorien. Anzahl und prozentualer } \\
\text { Anteil sind als } n(\%) \text { ausgewiesen. MW stellt den Mittelwert dar, SD die Standardabweichung und } \\
95 \%-K l \text { das } 95 \% \text {-Konfidenzintervall der Mittelwerte. Min Minimum, Max Maximum. Gruppenunter- } \\
\text { schiede wurden mittels ANOVA mit Bonferroni-Post-hoc-Test ermittelt. Chancenverhältnisse (Odds } \\
\text { ratio) mit zugehörigen Konfidenzintervallen und Bonferroni-korrigierte Signifikanzniveau ( } p \text { ) für } \\
\text { die Erfolgsraten, abhängig von der Punktionserfahrung in den } 3 \text { Kategorien. } p<0,05 \text { in allen Tests } \\
\text { statistisch signifikant (fett) }\end{array}$} \\
\hline
\end{tabular}

Die Gesamtrate an Pneumothoraces in den 3 Kategorien (0-20: 1,3\%, 21-50: 2,5\%, >50: 0,3\%; gesamte Population $2,2 \%)$ liegt im üblichen Bereich, der auch von anderen Autoren berichtet wurde $[6,19]$.

Mit steigender Zahl an Punktionsversuchen treten nachweislich auch mehr Komplikationen auf $[13,16,18]$. Daher wird gefordert, dass die Anzahl der Punktionen auf maximal 3 beschränkt werden soll und danach ein Verfahrenswechsel oder Austausch des Punktierenden stattfinden soll $[18,32]$. In dieser Studie lag die Anzahl der Punktionen in allen 3 Gruppen zwischen 1,6 und 1,9 im Mittel. Auch die Gesamtkomplikationsraten fallen relativ niedrig aus. Erklärungen hierfür können die vorgegebene und standardisierte Punktionstechnik sowie die Auswahl der Patienten sein. Die Patienten verfügten über einen grenzwertig normalen BMI, hatten keine Einschränkung der Blutgerinnung und verfügten über eine Normovolämie. Insgesamt also optimale Bedingungen, die die Interpretation unserer Ergebnisse einschränkt. Alle Punktionen wurden im elektiven Tagesprogramm durchgeführt und weisen dadurch per se eine niedrigere Komplikationsrate auf [20]. Des Weiteren wurden die unerfahrenen Punkteure grundsätzlich durch erfahrenere Ärzte/Ärztinnen supervidiert, wodurch diez. T. geringeren Komplikationsraten in der unerfahrensten Gruppe gegenüber der Gruppe mit 21 bis 50 Punktionen zu erklären wäre.

Die häufigste Komplikation bei der Anlage des VSK in unserer Studie war die Punktion der A. subclavia. Im gesamten Kollektiv trat die arterielle Fehlpunktion bei 5,7\% der Patienten auf und entspricht den Ergebnissen von anderen Autoren [7, 19, 20]. Einen signifikanten Unterschied innerhalb der Erfahrungsgruppen (0-20: 7,5\%, 21-50: 4,5\% und $>50: 4,6 \%)$ konnte allerdings nicht nachgewiesen werden.

Die im Studienprotokoll vorab festgelegten Erfahrungsgruppen lassen zwar den Schluss zu, dass es mit der Punktionserfahrung eine signifikante Lernkurve bezüglich Punktionserfolg, Zahl der Punktionsversuche und die Gesamtkomplikationsrate gibt, nicht aber in den Einzelkomplikationen Pneumothorax und arterielle Fehlpunktion. Es kann somit nicht ausgeschlossen werden, dass durch die Wahl anderer Erfahrungsgruppen in z.B. 10er-Schritten wie von Merrer et al. [20] ein präziserer und ggf. früherer „cut off“ für einzelne End- punkte hätte gefunden werden können. Betrachtet man jedoch die Subgruppe der Erstpunktierenden bezüglich des Punktionserfolges alleine (wenn nach 3 Punktionen kein Erfolg erreicht wurde, ist die Punktion als nichterfolgreich gewertet), so zeigt sich dieser Lernkurveneffekt noch deutlicher ab. Trotz Supervision schneidet die Gruppe 0-20 signifikant schlechter ab als die beiden erfahreneren Gruppen. Auch beim Vergleich zwischen den Gruppen 21-50 und mehr als 50 VSK ist die erfahrenere Gruppe signifikant besser, sodass bezüglich des Erfolges eine Lernkurve bis mindestens 50 Punktionen konstatiert werden kann.

Neben den bereits diskutierten gibt es weitere Limitationen in dieser Studie. Die hier untersuchte Anwendung der landmarkengestützten Punktion lässt eine Übertragung unserer Ergebnisse auf die ultraschallgestützte Punktion nicht zu. Die landmarkengestützte Punktion war zum Studienbeginn (2014) in beiden Studienzentren noch der Standard. Durch die mittlerweile ausreichend vorhandene Anzahl an Ultraschallgeräten hat in beiden Kliniken jedoch die Sonographie zur Anlage des VSK als Standard Einzug gefunden. Aufgrund der Ergebnisse zweier Studien [10, 23] empfehlen sowohl die ESA als auch die ASA [2, 14] heute zur Anlage eines VSK zumindest eine sonographische Vorabuntersuchung der Punktionsstelle zur Lokalisation und zur Beurteilung der Gefäße. Idealerweise sollte die Anlage unter Echtzeitsonographie stattfinden.

\section{Fazit für die Praxis}

- In dieser Post-hoc-Auswertung konnte gezeigt werden, dass in einem standardisierten Setting mit Supervision die Gesamtkomplikationsraten einer landmarkengestützten Punktionstechnik sehr gering sein können und eine Lernkurve mit signifikanter Verbesserung nach 50 Punktionen erreicht wird. Die Anzahl an Punktionsversuchen sollte 3 nicht überschreiten, und es muss dann großzügig der Punkteur ausgetauscht oder das Verfahren gewechselt werden. 


\section{- Nichtsdestotrotz ist die Echtzeit- Ultraschall-gestützte Punktion im elektiven Umfeld immer die erste Wahl. Gleichsam sollte die landmar- kengestützte Punktion erlernt und im Repertoire der Ärzte/Ärztinnen weiter vorhanden sein.}

\section{Korrespondenzadresse}

Dr.med. Thomas Palmaers

Klinik für Anästhesiologie und Intensivmedizin (OE8050), Medizinische Hochschule Hannover Carl-Neuberg-Str. 1, 30625 Hannover, Deutschland

palmaers.thomas@mh-hannover.de

Funding. Open Access funding enabled and organized by Projekt DEAL.

\section{Einhaltung ethischer Richtlinien}

Interessenkonflikt. J.Schulz, A. Scholler,P. Frank, D. Scheinichen, M. Flentje, H. Eismann und T. Palmaers geben an, dass kein Interessenkonflikt besteht.

Alle beschriebenen Untersuchungen am Menschen oder an menschlichem Gewebe wurden mit Zustimmung der zuständigen Ethikkommission, im Einklang mit nationalem Recht sowie gemäß der Deklaration von Helsinki von 1975 (in der aktuellen, überarbeiteten Fassung) durchgeführt. Von allen beteiligten Patienten liegt eine Einverständniserklärung vor.

Open Access. Dieser Artikel wird unter der Creative Commons Namensnennung 4.0 International Lizenz veröffentlicht, welche die Nutzung, Vervielfältigung, Bearbeitung, Verbreitung und Wiedergabe in jeglichem Medium und Format erlaubt, sofern Sie den/die ursprünglichen Autor(en) und die Quelle ordnungsgemäß nennen, einen Link zur Creative Commons Lizenz beifügen und angeben, ob Änderungen vorgenommen wurden.

Die in diesem Artikel enthaltenen Bilder und sonstiges Drittmaterial unterliegen ebenfalls der genannten Creative Commons Lizenz, sofern sich aus der Abbildungslegende nichts anderes ergibt. Sofern das betreffende Material nicht unter der genannten Creative Commons Lizenz steht und die betreffende Handlung nicht nach gesetzlichen Vorschriften erlaubt ist, ist für die oben aufgeführten Weiterverwendungen des $\mathrm{Ma}$ terials die Einwilligung des jeweiligen Rechteinhabers einzuholen.

Weitere Details zur Lizenz entnehmen Sie bitte der Lizenzinformation auf http://creativecommons.org/ licenses/by/4.0/deed.de.

\section{Literatur}

1. AmirR, KnioZO, MahmoodFetal (2017) Ultrasound as a screening tool for central venous catheter positioning and exclusion of pneumothorax. Crit Care Med 45:1192-1198
2. American Society of Anesthesiologists (2020) Practice guidelines for central venous access 2020: an updated report by the American Society of Anesthesiologists Task Force on Central Venous Access. Anesthesiology 132:8-43

3. Bell J, Goyal M, Long S et al (2020) Anatomic site-specific complication rates for central venous catheter insertions. J Intensive Care Med 35(9):869-874

4. Bjorkander M, Bentzer P, Schott U et al (2019) Mechanical complications of central venous catheter insertions: a retrospective multicenter study of incidence and risks. Acta Anaesthesiol Scand 63:61-68

5. Brass $P$, Hellmich M, Kolodziej L et al (2015) Ultrasound guidanceversus anatomicallandmarks for subclavian or femoral vein catheterization. Cochrane Database Syst Rev 1:CD11447

6. Chui J, Saeed R, Jakobowski Let al (2018) Is routine chestX-ray afterultrasound-guided central venous catheter insertion choosing wisely?: A populationbased retrospective study of 6,875 patients. Chest 154:148-156

7. Comerlato PH, Rebelatto TF, Santiago De Almeida FA et al (2017) Complications of central venous catheter insertion in a teaching hospital. Rev Assoc Med Bras 63:613-620

8. Eerola R, Kaukinen L, Kaukinen S (1985) Analysis of 13800 subclavian vein catheterizations. Acta Anaesthesiol Scand 29:193-197

9. Fosh B, Canepa M, Eaton M (2016) Long-term venous access insertion: "the learning curve". ANZJ Surg 86:1038-1041

10. Fragou M, Gravvanis A, Dimitriou V et al (2011) Real-time ultrasound-guided subclavian vein cannulation versus the landmark method in critical care patients: a prospective randomized study. Crit Care Med 39:1607-1612

11. Frykholm P, Pikwer A, Hammarskjöld F et al (2014) Clinical guidelines on central venous catheterisation. Swedish Society of Anaesthesiology and Intensive Care Medicine. Acta Anaesthesiol Scand 58:508-524

12. Iwanczuk W, Guzniczak P, Kasperczak J (2013) Hemothorax as a complication of subclavian vein cannulation with haemodialysis catheter-case report. Anaesthesiol Intensive Ther 45:89-92

13. Kilbourne MJ, Bochicchio GV, Scalea T et al (2009) Avoiding common technical errors in subclavian central venous catheter placement. J Am Coll Surg 208:104-109

14. Lamperti M, Biasucci DG, Disma N et al (2020) European Society of Anaesthesiology guidelines on peri-operative use of ultrasound-guided for vascular access (PERSEUS vascular access). Eur J Anaesthesiol 37:344-376

15. Lamperti M, Bodenham AR, Pittiruti M et al (2012) International evidence-based recommendations on ultrasound-guided vascular access. Intensive Care Med 38:1105-1117

16. Lefrant JY, Muller L, De La Coussaye JE et al (2002) Risk factors of failure and immediate complication of subclavian vein catheterization in critically ill patients. Intensive Care Med 28:1036-1041

17. Maizel J, Guyomarc'h L, Henon P et al (2014) Residents learning ultrasound-guided catheterization are not sufficiently skilled to use landmarks. Crit Care 18:R36

18. Mansfield PF, Hohn DC, Fornage BD et al (1994) Complications and failures of subclavian-vein catheterization. N Engl J Med 331:1735-1738

19. McGee DC, Gould MK (2003) Preventing complications of central venous catheterization. $\mathrm{N}$ Engl J Med348:1123-1133
20. Merrer J, De Jonghe B, Golliot F et al (2001) Complications of femoral and subclavian venous catheterization in critically ill patients: a randomized controlled trial. JAMA 286:700-707

21. National Institute for Clinical Excellence (2002) Guidance on the use of ultrasound locating devices for placing central venous catheters. NICE Technology Apraisal No. 49

22. Odendaal J, Kong VY, Sartorius B et al (2017) Mechanical complications of central venous catheterisation in trauma patients. Ann R Coll Surg Engl 99:390-393

23. Oh AY, Jeon YT, Choi EJ et al (2014) The influence of the direction of J-tip on the placement of a subclavian catheter: real time ultrasoundguided cannulation versus landmark method, a randomized controlled trial. BMC Anesthesiol 14:11

24. Omid M, Rafiei MH, Hosseinpour M et al (2015) Ultrasound-guided percutaneous central venous catheterization in infants: learning curve and related complications. Adv Biomed Res 4:199

25. Palmaers T, Frank P, Eismann H et al (2019) Catheterization of the subclavian vein and the risk of pneumothorax: mechanical ventilation increases the risk of pneumothorax during infraclavicular landmark-guided subclavian vein puncture: a prospective randomized study. Anaesthesist 68:309-316

26. Parienti JJ, Mongardon N, Megarbane B et al (2015) Intravascular complications of central venous catheterization by insertion site. N Engl J Med 373:1220-1229

27. Ruesch S, Walder B, Tramer MR (2002) Complications of central venous catheters: internal jugular versus subclavian access-a systematic review. Crit Care Med 30:454-460

28. Rupp SM, Apfelbaum JL, Blitt Cet al (2012) Practice guidelines for central venous access: a report by the American Society of Anesthesiologists Task Force on Central Venous Access. Anesthesiology 116:539-573

29. Seldinger SI (1953) Catheter replacement of the needle in percutaneous arteriography; a new technique. Acta Radiol 39:368-376

30. Soni NJ, Reyes LF, Keyt H et al (2016) Use of ultrasound guidance for central venous catheterization: a national survey of intensivists and hospitalists. J Crit Care 36:277-283

31. Sznajder Jl, Zveibil FR, Bitterman H et al (1986) Central vein catheterization. Failure and complication rates by three percutaneous approaches. Arch Intern Med 146:259-261

32. Takeyama H, Taniguchi M, Sawai H et al (2006) Limiting vein puncture to three needle passes in subclavian vein catheterization by the infraclavicular approach. Surg Today 36:779-782 